03

\title{
Взаимодействие интенсивных предельно коротких импульсов с квантовыми объектами
}

\author{
(C) Н.Н. Розанов \\ Государственный оптический институт им. С.И. Вавилова, \\ 199053 Санкт-Петербург, Россия \\ Университет ИТМО, \\ 197101 Санкт-Петербург, Россия \\ Физико-технический институт имени А.Ф.Иофффе, \\ 194021 Санкт-Петербург, Россия \\ e-mail: nnrosanov@mail.ru
}

Поступила в редакцию 02.08.2017 г.

\begin{abstract}
Предложено простое описание воздействия предельно интенсивного и короткого импульса излучения на квантовый объект. Общее приближенное решение нестационарного уравнения Шредингера конкретизируется для случая электродипольных переходов в атоме водорода.
\end{abstract}

DOI: $10.21883 /$ OS.2018.01.45361.174-17

\section{1. Введение}

Наиболее высокая концентрация энергии излучения достигается при использовании импульсов с предельно высокой пиковой интенсивностью [1] и предельно короткой длительностью [2-4]. Два этих направления принадлежат к числу наиболее стремительно развивающихся в современной лазерной физике и технике. В лазерных импульсах достигается электрическая напряженность поля $\mathbf{E}$, значительно превосходящая внутриатомную, а длительность импульсов, генерируемых сложением гармоник лазерного излучения, доходит до аттосекундного диапазона [5], что позволяет диагностировать сверхбыстрые процессы, ускорять заряженные частицы и т.д. Значительный интерес вызывает возможность формирования импульсов с высокой степенью униполярности [6].

$$
\xi=\frac{\left|\int \mathbf{E} d t\right|}{\int|\mathbf{E}| d t} \sim 1 .
$$

Отметим, что в числителе дроби в (1) фигурирует электрическая площадь импульса

$$
\mathbf{S}_{E}=\int \mathbf{E} d t
$$

обладающая интересными свойствами сохранения [7,8]. Поскольку в униполярных импульсах достигается однонаправленное воздействие поля на заряд, они обеспечивают более эффективное управление его динамикой по сравнению с биполярными импульсами.

Поскольку спектр предельно коротких импульсов весьма широк и может захватывать большое число даже электронных переходов, простые двухуровневые или малоуровневые модели взаимодействующих с излучением объектов оказываются недостаточными. С другой стороны, понятие резонансных переходов также оказывается неприменимым ввиду краткости импульса и соответственно малого времени раскачки отвечающих объекту осцилляторов. В этих условиях лучшее описание взаимодействия реализуется при численном решении нестационарного уравнения Шредингера для волновой функции объекта [9]. Задачей данного сообщения служит анализ простой модели взаимодействия квантового объекта (для определенности атома) с предельно интенсивным и коротким импульсом излучения, в которой не используется ограничение числа уровней. В определенном смысле здесь имеется аналогия с резким „встряхиванием“ атома под действием кратковременного возмущения $[10,11]$. Далее мы приводим приближенное решение нестационарного уравнения Шредингера, при выводе которого используются обе особенности обсуждаемых импульсов (высокие интенсивности и малая длительность). Затем это решение конкретизируется применительно к атому водорода. Общее обсуждение результатов содержится в Заключении.

\section{2. Динамика волновой функции}

Используя атомные единицы, исходим из нерелятивистского уравнения Шредингера для волновой функции $\Psi$

$$
i \frac{\partial \Psi}{\partial t}=H_{0} \Psi+V(\mathbf{r}, t) \Psi .
$$

Здесь $t$ и $\mathbf{r}$ - безразмерные время и радиус-вектор, $H_{0}$ - гамильтониан атомной системы и $V(\mathbf{r}, t)-$ потенциал взаимодействия атома с электромагнитным излучением. В отсутствие излучения волновая функция разлагается по собственным функциям энергетического спектра

$$
\Psi=\sum_{n} a_{n} \psi_{n}(r) \exp \left(-i E_{n} t\right)
$$

причем

$$
H_{0} \psi_{n}=E_{n} \psi_{n}, \quad a_{n}=\mathrm{const}, \quad \sum_{n}\left|a_{n}\right|^{2}=1 .
$$

В (4) и (5) подразумевается суммирование по дискретному спектру и интегрирование по сплошному. 
Полагаем, что электромагнитное излучение представляет импульс, интенсивный (по сравнению с кулоновским полем ядра, возможно, экранированным внутренней электронной оболочкой) и короткий по сравнению с обратными частотами $1 / E_{n}$. Тогда во время действия импульса основным в правой части (3) является последний член, и волновая функция после окончания импульса $\Psi_{t=+0}$ выражается через волновую функцию до начала импульса $\Psi_{t=-0}$ следующим образом:

$$
\Psi_{t=+0}=\exp \left(-i \int V d t\right) \Psi_{t=-0} .
$$

Интегрирование в (6) выполняется по всей длительности импульса. Если пространственно-временная зависимость потенциала взаимодействия факторизуется, $V(\mathbf{r}, t)=V_{r}(\mathbf{r}) V_{t}(t)$, как это имеет место для электродипольных переходов, то

$$
\Psi_{t=+0}=\exp \left(-i V_{r}(\mathbf{r}) \int V_{t}(t) d t\right) \Psi_{t=-0} .
$$

Поэтому атом сохраняет свое состояние, $\Psi_{t=+0}=\Psi_{t=-0}$, после взаимодействия с импульсом, у которого

$$
\int V_{t}(t) d t=0
$$

После окончания импульса волновая функция имеет вид (4) $[6$ с амплитудами состояний

$$
\begin{aligned}
a_{n} & =\left\langle\psi_{n} \mid \Psi_{t=+0}\right\rangle=\int \psi_{n}^{*} \Psi_{t=+0} d \mathbf{r} \\
& =\int \psi_{n}^{*}(\mathbf{r}) \exp \left(-\frac{i}{\hbar} \int V(\mathbf{r}, t) d t\right) \Psi_{t=-0}(\mathbf{r}) d \mathbf{r} .
\end{aligned}
$$

\section{3. Атом водорода}

Для атомов водорода или водородоподобных атомов спектр и волновые функции в отсутствие излучения находятся как точные решения стационарного уравнения Шредингера (5) для единственного электрона в кулоновском поле [10]. Будем считать, что до воздействия импульса атом находился в основном состоянии:

$$
\psi_{0}=\frac{1}{\sqrt{\pi}} e^{-r}
$$

Здесь $r=|\mathbf{r}|$. Потенциал взаимодействия электрона с излучением в электродипольном приближении (размеры атома много меньше характерных длин волн излучения) $V=\mathbf{E r}$, где $\mathbf{E}-$ электрическая напряженность поля. Соответственно

$$
\int V(\mathbf{r}, t) d t=\int \mathbf{E}(t) \mathbf{r} d t=\mathbf{S}_{E} \mathbf{r}=S_{E} z
$$

где $\mathbf{S}_{E}$ - электрическая площадь импульса (2) и ось $z$ направлена вдоль вектора $\mathbf{S}_{E}$. Теперь амплитуда основного состояния после окончания импульса

$$
a_{0}=\int \exp \left(-i S_{E} z\right) \psi_{0}^{2}(r) d \mathbf{r}=\frac{1}{\pi} \int \exp \left(-i S_{E} z\right) e^{-2 r} d \mathbf{r} .
$$

Интеграл вычисляется при использовании параболических координат, в которых [10]

$$
r=\frac{1}{2}(\xi+\eta), \quad z=\frac{1}{2}(\xi-\eta), \quad 0<\xi, \quad \eta<\infty .
$$

В результате находим

$$
a_{0}=\frac{1}{\left(1+\frac{S_{E}^{2}}{4}\right)^{2}}
$$

Соответственно вероятность сохранения основного состояния атома после действия одиночного импульса

$$
w_{0}=a_{0}^{2}=\frac{1}{\left(1+\frac{S_{E}^{2}}{4}\right)^{2}} .
$$

Вероятность $w_{0}$ монотонно убывает с ростом электрической площади импульса $S_{E}$ от 1 при $S_{E}=0$, принимая значения $w_{0}=0.41$ при $S_{E}=1,0.063$ при $S_{E}=2$ и 0.009 при $S_{E}=3$. Для импульсов с малой по сравнению с единицей электрической площадью $w_{0}=1-S_{E}^{2}$, а при большой площади $w_{0}=\left(2 / S_{E}\right)^{8}$. Аналогичным образом вычисляются вероятности возбуждения и ионизации атома.

Приведенный подход можно рассматривать как низшее приближение теории возмущений для уравнения (3), причем в качестве возмущения выступает член с „невозмущенным“ гамильтонианом $H_{0}$. Полагая $\Psi=\Psi^{(0)}+\Psi^{(1)}+\ldots$, решение (3) в низшем приближении записываем, согласно (6), в виде (опускаем аргумент r)

$$
\Psi^{(0)}(t)=\exp \left(-i \int_{0}^{t} V d t\right) \Psi(t=0)
$$

Первое приближение определяется уравнением

$$
i \frac{\partial \Psi^{(1)}}{\partial t}-V(t) \Psi^{(1)}=H_{0} \Psi^{(0)}(t)
$$

Решение (17)

$$
\begin{aligned}
\Psi^{(1)}(t)= & -i \exp \left(-i \int_{0}^{t} V d t^{\prime}\right) \\
& \times \int_{0}^{t} \exp \left(i \int_{0}^{t^{\prime}} V d t^{\prime \prime}\right) H_{0} \Psi^{(0)}\left(t^{\prime}\right) d t^{\prime}
\end{aligned}
$$

уже учитывает, в отличие от низшего приближения, искажения пространственного распределения вероятности нахождения электрона под действием импульса излучения. 


\section{4. Заключение}

Таким образом, удается получить простое выражение, описывающее вероятность возбуждения (и ионизации) квантового объекта (атома) под действием предельно коротких и интенсивных импульсов излучения. Существенно, что эта вероятность определяется в низшем приближении исключительно электрической площадью импульса - интегралом от напряженности электрического поля по всей длительности импульса. Естественно, что рассмотрение на основе уравнения Шредингера ограничено временами жизни возбужденных уровней. Отметим также, что использование предельно коротких - аттосекундных - импульсов могло бы позволить наблюдение динамики волновых пакетов (электронного облака) не только в молекулах, но и в атомах.

Работа поддержана грантом РФФИ № 16-02-00762_а.

\section{Список литературы}

[1] Электронный ресурс. Режим доступа: https://eli-laser.eu/

[2] Krausz F., Ivanov M. // Rev. Mod. Phys. 2009. V. 81. N 1. P. 163-234.

[3] Calegari F., Sansone G., Stagira S., Vozzi C., Nisoli M. // J. Phys. B: At. Mol. Opt. Phys. 2016. V. 49. N 6. P. 062001.

[4] Manzoni C., Mucke O.D., Cirmi G., Fang S., Moses J., Huang S.-W., Hong K.-H., Cerullo G., Kartner F.X. // Laser Photonics Rev. 2015. V. 9. N 2. P. 129-171.

[5] Ramasesha K., Leone S.R., Neumark D.M. // Annu. Rev. Phys. Chem. 2016. V. 67. P. 41-63.

[6] Архипов Р.М., Пахомов А.В., Архипов М.В., Бабушкин И., Толмачев Ю.А., Розанов Н.Н. // Письма в ЖЭТФ. 2017. T. 105. № 6. C. 388; Arkhipov R.M., Pakhomov A.V., Arkhipov M.V., Babushkin I., Tolmachev Yu.A., Rosanov N.N. // JETP Letters. 2017. V. 105. N 6. P. 408.

[7] Розанов Н.Н. // Опт. и спектр. 2009. Т. 107. № 5. С. 761765; Rosanov N.N. Opt. Spectrosc. 2009. V. 107. N 5. P. 721725.

[8] Розанов Н.Н. Диссипативные оптические солитоны. От микро- к нано- и атто-. М.: Физматлит, 2011. 536 с.

[9] Calvert J.E., Han Xu, Palmer A.J., Glover R.D., Laban D.E., Tong X.M., Kheifets A.S., Bartschat K., Litvinyuk I.V., Kielpinski D., Sang R.T. // Sci. Rep. 2016. V. 6. P. 34101.

[10] Ландау Л.Д., Либиии, Е.M. Квантовая механика. Нерелятивистская теория. М.: Наука, 1974. 752 с.

[11] Мигдал А.Б., Крайнов В.П. Приближенные методы квантовой механики. М.: Наука, 1966. 152 с. 